\title{
Factors limiting the utility of bronchoalveolar lavage in the diagnosis of COVID-19
}

\author{
To the Editor:
}

We read with interest study by GERI et al. [1], in which the authors have demonstrated a strong agreement between negative nasopharyngeal (NPs)/nasal (Ns) swab and bronchoalveolar lavage (BAL) real-time reverse transcriptase-polymerase chain reaction (rRT-PCR) in the diagnosis of coronavirus disease 2019 (COVID-19) among hospitalised patients. The study findings contradicted earlier reports [2] and suggested a limited utility of BAL. However, the results need to be interpreted comprehensively before drawing any conclusion.

In the present study, BAL was negative for severe acute respiratory syndrome coronavirus 2 (SARS-CoV-2) by rRT-PCR in the majority of cases, which included 38 patients (48\%) with a strong clinical and radiological suspicion for COVID-19. This finding implies either a high false-negative rate of BAL rRT-PCR or an alternate diagnosis. Hence, the authors should give a detailed account of the final diagnoses and treatment outcomes of the patients and correlate these with the rRT-PCR results. This will give a better picture of the clinical performance of rRT-PCR in both BAL and upper respiratory samples.

Clinical test performance of rRT-PCR (in BAL/Ns/NPs) is a dynamic parameter that depends not only on its analytical sensitivity but also on the pretest probability. The pretest probability may in turn depend on the SARS-CoV-2 exposure history, disease symptoms and local disease prevalence [3]. The unexpected low positivity seen with BAL rRT-PCR in the study, despite high pretest probability, might be due to delayed time of sampling and/or disease stage. All these factors should have been considered while comparing the diagnostic yield in the study.

BAL has a specific place in the diagnostic algorithm of COVID-19 and is usually performed in a patient with lower respiratory tract involvement and high clinical suspicion but negative Ns/NPs result. However, negative BAL results should be interpreted comprehensively in light of different clinical and demographic factors on a case-to-case basis.

@ERSpublications

Bronchoalveolar lavage has a specific place in the diagnosis of COVID-19. The clinical performance of BAL rRt-PCR is dynamic in nature and depends on different clinical and demographic factors. https:// bit.ly/3aZUAPB

Cite this article as: Aggarwal D, Saini V. Factors limiting the utility of bronchoalveolar lavage in the diagnosis of COVID-19. Eur Respir J 2020; 56: 2003116 [https://doi.org/10.1183/13993003.03116-2020].

Deepak Aggarwal and Varinder Saini

Dept of Pulmonary Medicine, Government Medical College and Hospital, Chandigarh, India.

Correspondence: Deepak Aggarwal, Dept of Pulmonary Medicine, Block-D, Level-5, Government Medical College and Hospital, Sector-32, Chandigarh, Pin-160030, India. E-mail: drdeepak@hotmail.com

Received: 12 Aug 2020 | Accepted: 20 Aug 2020

Conflict of interest: None declared.

\section{References}

1 Geri P, Salton F, Zuccatosta L, et al. Limited role for bronchoalveolar lavage to exclude COVID-19 after negative upper respiratory tract swabs: a multicentre study. Eur Respir J 2020; 56: 2001733.

2 Wang W, Xu Y, Gao R, et al. Detection of SARS-CoV-2 in different types of clinical specimens. JAMA 2020; 323: 1843-1844.

3 Woloshin S, Patel N, Kesselheim AS. False negative tests for SARS-CoV-2 infection - challenges and implications N Engl J Med 2020; 383: e38. 